\title{
Revisit of non-linear Landau damping for electrostatic instability driven by blazar-induced pair beams
}

\author{
Martin Pohl* \\ 1 University of Potsdam, Institute for Physics and Astronomy, University of Potsdam, D-14476 \\ Potsdam, Germany \\ 2 DESY, Platanenallee 6, D-15738 Zeuthen, Germany \\ E-mail: marpohl@uni-potsdam.de
}

\section{Pranab Deka and Sergei Vafin}

University of Potsdam, Institute for Physics and Astronomy, D-14476 Potsdam, Germany

\begin{abstract}
We revisit the effect of non-linear Landau damping on the electrostatic instability of blazarinduced pair beams, building on an earlier study of electrostatic-wave growth rates calculated for realistic pair-beam distributions. The new aspect in this paper is a simplified 2D model in $\mathbf{k}$-space that is used to study the evolution of the electric-field spectrum and to calculate the relaxation time of the beam. We verified that the $2 \mathrm{D}$ model is an adequate representation of the 3D physics. We find that non-linear Landau damping, once it operates efficiently, transports essentially the entire wave energy to small wavenumbers where wave driving is weak or absent. Formally, the relaxation time of the pair beam then is longer than the inverse Compton scattering time. We added collisions as a subdominant damping process for the waves and found that it reduces the wave intensity at very small $k$. Consequently, non-linear Landau damping is less efficient and the relaxation time of the pair beam reduced, albeit not as much as to be less than the inverse-Compton loss time. Any other loss process will act similarly, and a full description of the spectral evolution of the electrostatic waves is crucial for calculating the relaxation time of the pair beam.
\end{abstract}

36th International Cosmic Ray Conference -ICRC2019-

July 24th - August 1st, 2019

Madison, WI, U.S.A.

\footnotetext{
* Speaker.
} 


\section{Introduction}

The propagation of very high energy gamma-radiation $\left(E_{\gamma}>100 \mathrm{GeV}\right)$ and its absorption in the inter-galactic medium (IGM) have been actively studied in recent years both observationally $[13,14,1,9,7,10]$ and theoretically $[8,23,3,15,17,18,12,20,4,16,24]$. The high-energy photons interact with the extra-galactic background light (EBL), producing ultra-relativistic electronpositron beams [11], that emit secondary photons [2]. In some cases the measured gamma-ray signal in the GeV energy band is smaller than that predicted assuming that the pairs lose their energy only due to the IC scattering [14, 21, 22]. Thus, some other dissipation processes must be in play.

One may posit that the absence of the cascade signal arises from magnetic deflection, which would require fG-scale magnetic field in cosmic voids [8, 14, 23]. In that case one would expect to see deflected cascade emission from radio galaxies which, however, has also not been seen [5].

An alternative model is based on energy loss that arises as the electron-positron beam propagating through the IGM plasma drives the electrostatic (two-stream) instability [3, 18, 24]. Direct simulations of the instability are difficult, because real beams require an excessive grid resolution [19], and modified beam parameters need to be carefully selected, otherwise unwanted instabilities or other issues can impair the simulation [16]. Analytical theory provides the linear growth rate of the waves, $\omega_{i}$, and one finds that for realistic beam parameters obliquely propagating waves experience the fastest growth, although also waves propagating parallel to the beam or perpendicular to the beam can grow [24].

Once one knows the spectral energy density of the electrostatic waves, $W_{k}$, one can calculate the driving power and hence the energy loss rate for the pair beam

$$
\dot{E}=-8 \pi \int \omega_{i} W\left(k_{\perp}, t\right) k_{\perp} d k_{\perp} d k_{\|} .
$$

It is obvious that knowledge of the saturation spectrum of the waves is essential. Previous studies either used a simplified model of non-linear damping process, assumed a steady-state wave spectrum after reaching saturation, or invoked an unrealistically large wave growth rates at very large $k_{\perp}$. The primary goal of this paper is to investigate the effect of non-linear Landau damping considering the realistic behavior of the electrostatic growth rate at large wave numbers for blazar-induced pair beams.

For a more detailed description of this research we refer to our main paper [25].

\section{Method}

The non-linear wave kinetic equation including driving (i), linear (LL) and non-linear (NL) Landau damping, as well as collisions (c) reads

$$
\frac{d W(\mathbf{k})}{d t}=2\left(\omega_{i}(\mathbf{k})+\omega_{L L}(\mathbf{k})+\omega_{N L}(\mathbf{k})+\omega_{c}\right) W(\mathbf{k}) .
$$

The growth rate, $\omega_{i}$, is taken from [24]. The damping rates are

$$
\omega_{L L}(\mathbf{k})=-\omega_{p, e} \sqrt{\frac{\pi}{8}}\left(\frac{\omega_{p, e}}{k u_{e}}\right)^{3} \exp \left[-\frac{1}{2}\left(\frac{\omega_{p, e}}{k u_{e}}\right)^{2}\right]
$$


and

$$
\omega_{N L}(\mathbf{k})=\frac{3(2 \pi)^{1 / 2}}{64 n_{e} m_{e} u_{i}} \int d^{3} k^{\prime} W\left(\mathbf{k}^{\prime}\right) \frac{\left(\mathbf{k} \mathbf{k}^{\prime}\right)^{2}}{\left(k^{\prime} k\right)^{2}} \frac{k^{\prime 2}-k^{2}}{\left|\mathbf{k}^{\prime}-\mathbf{k}\right|} \exp \left[-a\left(\frac{c}{\omega_{p, e}} \frac{k^{\prime 2}-k^{2}}{\left|\mathbf{k}^{\prime}-\mathbf{k}\right|}\right)^{2}\right] .
$$

Here, $a=(9 / 8)\left[u_{e}^{2} /\left(c u_{i}\right)\right]^{2}$, where $u_{i, e}=\sqrt{T_{\mathrm{IGM}} / m_{i, e}}$ denotes the thermal speeds of ions and electrons in the IGM, and $T_{\mathrm{IGM}}$ is the temperature. For $T_{\mathrm{IGM}}=10^{4} T_{4} \mathrm{~K}$ we find $a \simeq 3.6 \cdot 10^{-3} T_{4}$.

The collisional damping rate reads

$$
\omega_{c}=-1.45 \cdot 10^{-6} n_{e} \lambda T_{e}^{-3 / 2}\left[\mathrm{~s}^{-1}\right],
$$

where $\lambda=23.5-\ln \left(n_{e}^{1 / 2} T_{\mathrm{IGM}}^{-5 / 4}\right)-\left[10^{-5}+\left(\ln T_{\mathrm{IGM}}-2\right)^{2} / 16\right]^{1 / 2}$ is the Coulomb logarithm and $n_{e}$ and $T_{\mathrm{IGM}}$ are in units $\mathrm{cm}^{-3}$ and $\mathrm{eV}$, respectively. Moreover, we have implicitly assumed that the real part of the frequency $\omega$ is approximately equal to the plasma frequency, $\omega_{p, e}$. Likewise, we assume purely electrostatic waves with $\mathbf{k} \| \mathbf{E}$.

We calculated the pair beam spectrum at a distance $D=50 \mathrm{Mpc}$ from a token blazar with primary gamma-ray spectrum in the form of a power law with index $s=1.8$. Obviously the density of the pair beam will scale as $D^{-2}$, and so there will be stronger driving close to the blazar than far from it. The optical depth for primary gamma rays that spawn cascade emission in the $\mathrm{GeV}$ band is about $80 \mathrm{Mpc}$, and so we expect that our choice of distance reflects the location where the bulk of the cascade energy is processed.

As we explore the viability of dominant energy drain by plasma instabilities, we calculated the pair spectrum neglecting Compton cooling. This is an optimistic assumption, because Compton cooling will soften the spectrum and lead to a lower wave growth rate [12].

As the two-stream resonance is extremely narrow for a $\Gamma=10^{6}$ pair beam, very high resolution in $k$ space is required to numerically solve equation 2.1. To make things worse, the Compton energy loss rate is more than a million times weaker than the peak growth rate of wave that we need to resolve. Both conditions together make a numerical solution of equation 2.1 excessively difficult. We introduce two simplifications. First, azimuthal symmetry allows to write equations 2.1 and 2.3 in $k_{\perp}$ and $k_{\|}$, and the integration over the azimuthal angle, $\phi_{k}$, can be performed semi-analytically, as $W_{k}$ is independent of it.

In additions, we further reduce the dimensionality by using a 2D model $\left(W=W\left(k_{\perp}\right)\right.$ ) for analyzing the problem in the plane $k_{\|} c / \omega_{p, e} \approx 1$ of the $\mathbf{k}$-space. To preserve the fact that the waves are scattered out to the non-resonant wave vectors, i.e., out of the instability region, we modify the growth rate to

$$
\omega_{i}\left(k_{\perp}\right)=\frac{\omega_{i}\left(k_{\perp} c / \omega_{p, e}=1\right) \theta\left(k_{\perp} c / \omega_{p, e}-1\right)}{1+\left(k_{\perp} c / \omega_{p, e}\right)^{1.7}} \approx 5.6 \cdot 10^{-6} \frac{n_{b 20}}{\sqrt{n_{e 7}}} \frac{\theta\left(k_{\perp} c / \omega_{p, e}-1\right)}{1+\left(k_{\perp} c / \omega_{p, e}\right)^{1.7}}, \mathrm{~s}^{-1}
$$

where $\theta$ is the Heaviside step function. The growth rate is truncated at $k_{\perp} c / \omega_{p, e}=1$, and waves at small $k_{\perp}$ are assumed to be stable. The damping rates can be reduced to

$$
\omega_{L L}\left(k_{\perp}\right)=-\omega_{p, e} \sqrt{\frac{\pi}{8}}\left(\frac{\omega_{p, e}}{k_{\perp} u_{e}}\right)^{3} \exp \left[-\frac{1}{2}\left(\frac{\omega_{p, e}}{k_{\perp} u_{e}}\right)^{2}\right]
$$


and

$$
\omega_{N L}\left(k_{\perp}\right)=\frac{3(2 \pi)^{1 / 2}}{64 n_{e} m_{e} u_{i}} \int d^{2} k_{\perp}^{\prime} W\left(k_{\perp}^{\prime}, t\right) \frac{\left(\mathbf{k}_{\perp} \mathbf{k}_{\perp}^{\prime}\right)^{2}}{\left(k_{\perp}^{\prime} k_{\perp}\right)^{2}} \frac{k_{\perp}^{\prime 2}-k_{\perp}^{2}}{\left|\mathbf{k}_{\perp}^{\prime}-\mathbf{k}_{\perp}\right|} \exp \left[-a\left(\frac{c}{\omega_{p, e}} \frac{k_{\perp}^{\prime 2}-k_{\perp}^{2}}{\left|\mathbf{k}_{\perp}^{\prime}-\mathbf{k}_{\perp}\right|}\right)^{2}\right],
$$

and the total electrostatic energy density is

$$
W_{\mathrm{tot}}(t)=2 \pi \int W\left(k_{\perp}, t\right) k_{\perp} d k_{\perp} .
$$

Numerical and analytical tests show that this approximation preserves the essential characteristics of the system.

\section{Results}

For a full discussion of the results we refer to [25]. In the following summarize the main findings.

In contrast to an earlier study [24], who assumed that the wave spectrum at saturation is in a steady state, we find that quasi-perpendicular waves $\left(k_{\|} c / \omega_{p, e} \simeq 1\right.$ and $\left.k_{\perp} c / \omega_{p, e} \gg 1\right)$ display a complicated and time-variable spectrum whose intensity is typically too low to impose significant beam dissipation. In the spectral band where the growth rate is highest $\left(k_{\|} c / \omega_{p, e} \simeq 1\right.$ and $k_{\perp} c / \omega_{p, e} \approx 1$ ), the wave intensity is completely drained by NL damping driven by very high wave intensity at small $k$. Taken at face value, these results suggest that the beam dissipates a negligible portion of its energy during the IC cooling time. However, if other dissipation mechanisms are present that can efficiently dissipate the non-resonant waves at small $k$ that are responsible for a high NL damping rate, then the relaxation time of the beam would be significantly reduced. Among the possible candidates are the modulation instability and the collisional damping. In the current work, we considered only the latter.

Since the effect of collisions becomes important at small plasma temperatures, we performed several calculations with $T_{\mathrm{IGM}}<1 \mathrm{eV}$ including collisional damping. We found that the relaxation time is indeed considerably shorter than without collisions. This is surprising in view of the collision rate being about four orders of magnitude smaller than the peak growth rate of the electrostatic waves, and it reflects the delayed build-up of wave intensity at small $k$ by NL damping. Formally, even for $T_{\mathrm{IGM}}=0.3 \mathrm{eV}$, the relaxation time is still longer than the IC time. The large impact of a seemingly sub-dominant process indicates that the beam dissipation rate is extremely sensitive to the dissipation processes under consideration and to the accuracy of their treatment. The latter is best seen in the difference in estimated relaxation time between approximating NL damping by a simple decay term (as in [24]) and explicitly following the evolution of the wave spectrum, as done here. Thus, any other damping process (e.g., the modulation instability) will further modify the dissipation rate, and a full spectral treatment of all damping and cascading processes is needed to reliably calculate the beam dissipation rate, but that is a topic of future investigations.

The effect of collisional damping in the beam dissipation process is substantially small in the sense that the relaxation time is much larger than the IC scattering time even in their presence. Moreover, it is inversely dependent on the IGM temperature. In principle, for a sufficiently cold plasma, collisions could play a major role in beam energy dissipation, despite it being one of the 
slowest processes. Other processes like wave-wave scattering or wave-particle scattering produce higher rates thereby dominating the effect of particle collisions [6]. Other commonly ignored processes may play a role as well, and so an accurate estimate of the dissipation rate, and consequently, the relaxation time requires one to consider all possible damping mechanisms. Since collisional damping is not a predominant factor in the beam dissipation process, a direct constraint on the IGM temperature from its effect appears to be out of reach.

This paper demonstrates that theoretically calculating the beam dissipation rate is very challenging on account of the inherent non-linearity, and simple estimates may be misleading. It is conceivable that beam dissipation is efficient only for beams of a certain density, which translates to a maximum distance, $L_{\max }$, from the AGN that depends on its multi-TeV gamma-ray flux. We are not yet in the position to reliably calculate that distance and hence to estimate which part of the cascade would be quenched (at $L<L_{\max }$ ) and what spectrum the observable cascade produced at $L>L_{\max }$ would have.

\section{References}

[1] Ackermann, M., Ajello, M., Allafort, A., et al. 2012, Sci., 338, 1190

[2] Aharonian, F. A., Coppi, P. S., \& Voelk, H. J. 1994, ApJL, 423, L5

[3] Broderick, A. E., Chang, P., \& Pfrommer, C. 2012, ApJ, 752, 22

[4] Broderick, A. E., Tiede P., Shalaby, M. 2016, ApJ, 832, 109

[5] Broderick, A. E., Tiede, P., Chang, P., et al. 2018, ApJ 868, 87

[6] Chang, P., Broderick, A. E., Pfrommer, C., Puchwein, E., Lamberts, A., \& Shalaby, M. 2014, ApJ, 797,110

[7] de Naurois, M. 2015, Proceedings of the 34th International Cosmic Ray Conference, Netherlands

[8] Elyiv, A., Neronov, A., \& Semikoz, D. V. 2009, Phys. Rev. D, 80, 023010

[9] Frank, M. R., de Oña-Wilhelmi, E., \& Aharonian, F. A. 2013, Front. Phys., 8(6), 714

[10] Funk, S. 2015, Annu. Rev. Nucl. Part. Sci., 65, 245

[11] Gould, R. J., \& Schréder, S. 1966, PhRvL, 16, 748

[12] Miniati, F., \& Elyiv, A. 2013, ApJ, 770, 54

[13] Neronov, A., \& Semikoz, D. V. 2009, Phys. Rev. D, 80, 123012

[14] Neronov, A., \& Vovk, I. 2010, Science, 328, 73

[15] Puchwein, E., Pfrommer, C., Springel, V., Broderick, A. E., \& Chang, P. 2012, MNRAS, 423, 149

[16] Rafighi, I., Vafin, S., Pohl, M., \& Niemiec, J. 2017, A\&A, 607, A112

[17] Schlickeiser, R., Elyiv, A., Ibscher, D., \& Miniati, F. 2012a, ApJ, 758, 101

[18] Schlickeiser, R., Ibscher, D., \& Supsar, M. 2012b, ApJ, 758, 102

[19] Shalaby, M., Broderick, A. E., Chang, P., et al. 2018, ApJ, 859, 45

[20] Sironi, L., \& Giannios, D. 2014, ApJ, 787, 49

[21] Tavecchio, F., Ghisellini, G., Foschini, L., et al. 2010, MNRAS, 406, L70 
[22] Tavecchio, F., Ghisellini, G., Bonnoli, G., \& Foschini, L. 2011, MNRAS, 414, 3566

[23] Taylor, A. M., Vovk, I., \& Neronov, A. 2011, A\&A, 529, A144

[24] Vafin, S., Rafighi, I., \& Pohl, M. 2018, ApJ, 857, 43

[25] Vafin, S., Deka, P. J., Pohl, M., et al. 2019, ApJ, 873, 10 